\title{
ALGEBRO-GEOMETRIC SOLUTION OF THE DISCRETE KP EQUATION OVER A FINITE FIELD OUT OF A HYPERELLIPTIC CURVE
}

\author{
MARIUSZ BIAŁECKI AND ADAM DOLIWA
}

\begin{abstract}
We transfer the algebro-geometric method of construction of solutions of the discrete KP equation to the finite field case. We emphasize role of the Jacobian of the underlying algebraic curve in construction of the solutions. We illustrate in detail the procedure on example of a hyperelliptic curve.
\end{abstract}

\section{INTRODUCTION}

Cellular automata are dynamical systems on a lattice with values being discrete (usually finite) as well. They are one of the more popular and distinctive classes of models of complex systems. Introduced in various contexts [28, 23] around 1950 they have found wide applications in different areas, from physics through chemistry and biology to social sciences [29].

One of the most interesting properties of cellular automata is that complex patterns can emerge from very simple uptade rules. However, usually one cannot easily predict how a given cellular automaton will behave without going through a number of time steps on a computer. Due to their completely discrete nature, cellular automata are naturally suitable for computer simulations, but also here it would be instructive to have examples of rules with large classes of analytical solutions, integrals of motions and other "integrable features".

The problem of construction of integrable cellular automata is not new and was undertaken in a number of papers (see, for example, 8, 4, 5, 26]). In particular in [27, 18] it was given a systematic method, called ultra-discretization, of obtainig cellular automaton version of a given discrete integrable system.

Recently a new approach to integrable cellular automata was proposed in [7]. Its main idea is to keep the form of a given integrable discrete system but to transfer the algebro-geometric method of construction of its solutions [14, 1] from the complex field $\mathbb{C}$ to a finite field. This method, which in principle can be applied to any integrable discrete system with known algebro-geometric method of solution, has been applied to the fully discrete 2D Toda system (the Hirota equation) and in [3] to discrete KP and $\mathrm{KdV}$ equations (in Hirota form). In particular, the finite field valued multisoliton solutions of these equations have been constructed. We remark that the algebraic geometry over finite fields, although conceptually similar to that over the field of

2000 Mathematics Subject Classification. 14H70, 37K10, 37B15.

Key words and phrases. Integrable systems; cellular automata; finite fields; algebraic curves; discrete KP equation. 
complex numbers [10, has its own tools and peculiarities [6, 21]. It is also nowadays very important in practical use in modern approaches to public key cryptography [13] and in the theory of error correcting codes [25].

The aim of this paper is to study in the finite field context the very distinguished example of integrable system - the discrete KP equation. We present the algebrogeometric scheme of construction of its solutions in a finite field and we demonstrate its linearization on the level of the abstract Jacobi variety of the corresponding algebraic curve. We illustrate details of the construction on example of a hyperelliptic curve.

We remark that in [4] it was observed that the Lax representation of the discrete sine-Gordon equation of Hirota [11] has a meaning also when the field of complex numbers is replaced by a finite field of the form $\mathbb{F}_{p^{2}}$, where $p$ is a prime number. The authors of [4] showed also that, in principle, the corresponding integrals of motion can be calculated. Finally, we note that possibility of considering the soliton theory in positive characteristic has been anticipated in 22 .

The organization of the paper is as follows. In Section2 we first summarize the finite field version of Krichever's construction of solutions of the discrete KP equation, then we present its abstract Jacobian picture. In Section 3 we apply the method starting from an algebraic curve of genus two.

\section{The Finite Field SOlution of the Discrete KP EQUATion out of NONSINGULAR ALGEBRAIC CURVES}

We first briefly recall algebro-geometric construction of solutions of the discrete KP equation over finite fields [7, 3]. We discuss in addition a possible degeneracy of the linear problem and its consequences. Then we present the Jacobian picture of the construction, in which the integrable nature of the equation is evident. We point out some aspects of the representation which will help us to construct effectively solutions of the equation.

2.1. General construction. Consider an algebraic projective curve $\mathcal{C} / \mathbb{K}$ (or simply $\mathcal{C}$ ), absolutely irreducible, nonsingular, of genus $g$, defined over the finite field $\mathbb{K}=\mathbb{F}_{q}$ with $q$ elements, where $q$ is a power of a prime integer $p$ (see, for example [25, 10]). By $\mathcal{C}(\mathbb{K})$ we denote the set of $\mathbb{K}$-rational points of the curve. By $\overline{\mathbb{K}}$ denote the algebraic closure of $\mathbb{K}$, i.e., $\overline{\mathbb{K}}=\bigcup_{\ell=1}^{\infty} \mathbb{F}_{q^{\ell}}$, and by $\mathcal{C}(\overline{\mathbb{K}})$ denote the corresponding infinite set (often identified with $\mathcal{C}$ ) of $\overline{\mathbb{K}}$-rational points of the curve. The action of the Galois group $G(\overline{\mathbb{K}} / \mathbb{K}$ ) (of automorphisms of $\overline{\mathbb{K}}$ which are identity on $\mathbb{K}$, see [17]) extends naturally to action on $\mathcal{C}(\overline{\mathbb{K}})$.

Let us choose:

1. four points $A_{i} \in \mathcal{C}(\mathbb{K}), \quad i=0,1,2,3$,

2. effective $\mathbb{K}$-rational divisor of order $g$, i.e., $g$ points $B_{\gamma} \in \mathcal{C}(\overline{\mathbb{K}}), \gamma=1, \ldots, g$, which satisfy the following $\mathbb{K}$-rationality condition

$$
\forall \sigma \in G(\overline{\mathbb{K}} / \mathbb{K}), \quad \sigma\left(B_{\gamma}\right)=B_{\gamma^{\prime}}
$$

As a rule we assume here that all the points used in the construction are distinct and in general position. In particular, the divisor $\sum_{\gamma=1}^{g} B_{\gamma}$ is non-special. 
Definition 1. Fix $\mathbb{K}$-rational local parameter $t_{0}$ at $A_{0}$. For any integers $n_{1}, n_{2}, n_{3} \in$ $\mathbb{Z}$ define the function $\psi\left(n_{1}, n_{2}, n_{3}\right)$ as a rational function on the curve $\mathcal{C}$ with the following properties:

1. it has pole of the order at most $n_{1}+n_{2}+n_{3}$ at $A_{0}$,

2. the first nontrivial coefficient of its expansion in $t_{0}$ at $A_{0}$ is normalized to one,

3. it has zeros of order at least $n_{i}$ at $A_{i}$ for $i=1,2,3$,

4. it has at most simple poles at points $B_{\gamma}, \gamma=1, \ldots, g$.

As usual, zero (pole) of a negative order means pole (zero) of the corresponding positive order. Correspondingly one should exchange the expressions "at most" and "at least" in front of the orders of poles and zeros. By the standard (see e.g., [1]) application of the Riemann-Roch theorem (and the general position assumption) we conclude that the wave function $\psi\left(n_{1}, n_{2}, n_{3}\right)$ exists and is unique. The function $\psi\left(n_{1}, n_{2}, n_{3}\right)$ is $\mathbb{K}$-rational, which follows from $\mathbb{K}$-rationality conditions of sets of points in their definition.

Remark. In what follows we will often normalize functions in a sense of point 2 of Definition 1.

Fix $\mathbb{K}$-rational local parameters $t_{i}$ at $A_{i}, i=1,2,3$. In the generic case, which we assume in the sequel, when the order of the pole of $\psi\left(n_{1}, n_{2}, n_{3}\right)$ at $A_{0}$ is $n_{1}+n_{2}+n_{3}$ denote by $\zeta_{k}^{(i)}\left(n_{1}, n_{2}, n_{3}\right), i=0,1,2,3$, the $\mathbb{K}$-rational coefficients of expansion of $\psi\left(n_{1}, n_{2}, n_{3}\right)$ at $A_{i}$, respectively, i.e.,

$$
\begin{aligned}
& \psi\left(n_{1}, n_{2}, n_{3}\right)=\frac{1}{t_{0}^{\left(n_{1}+n_{2}+n_{3}\right)}}\left(1+\sum_{k=1}^{\infty} \zeta_{k}^{(0)}\left(n_{1}, n_{2}, n_{3}\right) t_{0}^{k}\right), \\
& \psi\left(n_{1}, n_{2}, n_{3}\right)=t_{i}^{n_{i}} \sum_{k=0}^{\infty} \zeta_{k}^{(i)}\left(n_{1}, n_{2}, n_{3}\right) t_{i}^{k}, \quad i=1,2,3 .
\end{aligned}
$$

Denote by $T_{i}$ the operator of translation in the variable $n_{i}, i=1,2,3$, for example $T_{1} \psi\left(n_{1}, n_{2}, n_{3}\right)=\psi\left(n_{1}+1, n_{2}, n_{3}\right)$. Uniqueness of the wave function implies the following statement.

Proposition 1. Generically, the function $\psi$ satisfies equations

$$
T_{i} \psi-T_{j} \psi+\frac{T_{j} \zeta_{0}^{(i)}}{\zeta_{0}^{(i)}} \psi=0, \quad i \neq j, \quad i, j=1,2,3 .
$$

Remark. When the genericity assumption fails then the linear problem (11) degenerates, i.e., some of its terms are absent.

Notice that equation (11) gives

$$
\frac{T_{j} \zeta_{0}^{(i)}}{\zeta_{0}^{(i)}}=-\frac{T_{i} \zeta_{0}^{(j)}}{\zeta_{0}^{(j)}}, \quad i \neq j, \quad i, j=1,2,3 .
$$

Define

$$
\rho_{i}=(-1)^{\sum_{j<i} n_{j}} \zeta_{0}^{(i)}, \quad i=1,2,3
$$


then equation (2) implies existence of a $\mathbb{K}$-valued potential (the $\tau$-function) defined (up to a multiplicative constant) by formulas

$$
\frac{T_{i} \tau}{\tau}=\rho_{i}, \quad i=1,2,3
$$

Finally, equations (11) give rise to condition

$$
\frac{T_{2} \rho_{1}}{\rho_{1}}-\frac{T_{3} \rho_{1}}{\rho_{1}}+\frac{T_{3} \rho_{2}}{\rho_{2}}=0
$$

which written in terms of the $\tau$-function gives the discrete KP equation [12] called also the Hirota equation

$$
\left(T_{1} \tau\right)\left(T_{2} T_{3} \tau\right)-\left(T_{2} \tau\right)\left(T_{3} T_{1} \tau\right)+\left(T_{3} \tau\right)\left(T_{1} T_{2} \tau\right)=0
$$

Corollary 2. Equation (5) can be obtained also from expansion of equation (11) at $A_{k}$, where $k=1,2,3, k \neq i, j$.

Remark. Absence of a term in the linear problem (11) (see the remark after Proposition 11) reflects, due to Corollary 2, in absence of the corresponding term in equation (6). This implies that in the non-generic case, when we have not defined the $\tau$-function yet, we are forced to put it to zero. Let us notice in advance (see the next section) that it is in complete analogy with the well known (complex field) interpretation of the algebro-geometric solution $\tau$ of the discrete KP equation as, essentially, the Riemann theta function.

2.2. The Jacobian interpretation. Denote by $\operatorname{Div}(\mathcal{C})$ the abelian group of the divisors on the curve $\mathcal{C}$ and by $\operatorname{Pic}^{0}(\mathcal{C})$ the group of eqivalence classes of degree zero divisors $\operatorname{Div}^{0}(\mathcal{C})$ modulo the principal divisors. There exists [16, 20, an abelian variety $J(\mathcal{C})$ of dimension $g$ (the Jacobian of the curve) and an injective map $\phi: \mathcal{C} \rightarrow J(\mathcal{C})$ (the Abel map) such that the extension of $\phi$ to $\operatorname{Div}(\mathcal{C})$ establishes an isomorphism between $\operatorname{Pic}^{0}(\mathcal{C})$ and $J(\mathcal{C})$. Moreover, if there exists a $\mathbb{K}$-rational point $A \in \mathcal{C}(\mathbb{K})$ of the curve, then $\phi$ can be defined by

$$
\mathcal{C} \ni P \mapsto \phi(P)=[P-A] \in J(\mathcal{C}),
$$

where $[P-A]$ designates the class of the degree zero divisor $P-A$ in $\operatorname{Pic}^{0}(\mathcal{C})$.

Denote by $\mathbb{D}_{r}(\mathcal{C})$ the effective divisors of degree $r$ of the curve $\mathcal{C}$ and by $\phi_{r}$ the extension of $\phi$ to $\mathbb{D}_{r}(\mathcal{C})$

$$
\mathbb{D}_{r}(\mathcal{C}) \ni D \mapsto \phi_{r}(D)=[D-r \cdot A] \in J(\mathcal{C}) .
$$

The direct image of $\phi_{r}$ is a subvariety $\mathbb{W}_{r}$ of dimension $r$ if $0 \leq r \leq g$, and of dimension $g$ if $r>g$. In particular, $\mathbb{W}_{g-1}$ defines a divisor in $J(\mathcal{C})$.

The group $\operatorname{Pic}^{0}(\mathcal{C} ; \mathbb{K})$ of eqivalence classes of $\mathbb{K}$-rational degree zero divisors $\operatorname{Div}^{0}(\mathcal{C} ; \mathbb{K})$ modulo the principal $\mathbb{K}$-rational divisors can be identified with the abelian group $J(\mathcal{C} ; \mathbb{K})$ of $\mathbb{K}$-rational points of the Jacobian variety. For finite field $\mathbb{K}$ the group $\operatorname{Pic}^{0}(\mathcal{C} ; \mathbb{K})$ is finite as well and its order can be found using properties of the zeta function of the curve (see, for example [25]). 
Let us present in this picture the description of the wave function $\psi$ and of the $\tau$ function. We choose the point $A_{0}$ as the reference point $A$ and consider the following divisor $D\left(n_{1}, n_{2}, n_{3}\right) \in \operatorname{Div}^{0}(\mathcal{C} ; \mathbb{K})$ of degree zero

$$
D\left(n_{1}, n_{2}, n_{3}\right)=n_{1}\left(A_{0}-A_{1}\right)+n_{2}\left(A_{0}-A_{2}\right)+n_{3}\left(A_{0}-A_{3}\right)+\sum_{\gamma=1}^{g} B_{\gamma}-g \cdot A_{0},
$$

with linear dependence on $n_{1}, n_{2}$ and $n_{3}$. Its equivalence class in $\operatorname{Pic}^{0}(\mathcal{C} ; \mathbb{K})$ has the unique $\mathbb{K}$-rational representant of the form

$$
X\left(n_{1}, n_{2}, n_{3}\right)=\sum_{\gamma=1}^{g} X_{\gamma}\left(n_{1}, n_{2}, n_{3}\right)-g \cdot A_{0} .
$$

This equivalence is given by a function whose divisor reads

$$
n_{1}\left(A_{1}-A_{0}\right)+n_{2}\left(A_{2}-A_{0}\right)+n_{3}\left(A_{3}-A_{0}\right)+\sum_{\gamma=1}^{g} X_{\gamma}\left(n_{1}, n_{2}, n_{3}\right)-\sum_{\gamma=1}^{g} B_{\gamma} \sim 0 .
$$

If we normalize such a function at $A_{0}$ according to Definition 1 it becomes the wave function $\psi$. Notice that some of $X_{\gamma}$ could be $A_{0}$ which would mean that $\left[D\left(n_{1}, n_{2}, n_{3}\right)\right] \in \mathbb{W}_{g-1}$. This correspondence gives rise to a set of important facts.

Corollary 3. Evolutions in variables $n_{1}, n_{2}$ and $n_{3}$ define linear flows in the Jacobian.

Corollary 4. Points $X_{\gamma}, \gamma=1, \ldots, g$ indicate zeros of the wave function which are not specified in the previous construction.

Corollary 5. If $\left[D\left(n_{1}, n_{2}, n_{3}\right)\right] \in \mathbb{W}_{g-1}$ then the pole of the wave function at $A_{0}$ has the order less then $\left(n_{1}+n_{2}+n_{3}\right)$, i.e., we are in the non-generic case, thus $\tau\left(n_{1}, n_{2}, n_{3}\right)=0$.

Remark. Notice that because $[D(0,0,0)] \notin \mathbb{W}_{g-1}$ then $\tau(0,0,0) \neq 0$.

Remark. If $\mathbb{K}=\mathbb{C}$ then the algebraic curve $\mathcal{C}$ is the compact Riemann surface, theorems of Abel and Jacobi identify the Jacobian with quotient of $\mathbb{C}^{g}$ by the period lattice, and a theorem of Riemann identifies $\mathbb{W}_{g-1}$ with a certain translate of the zero locus of the Riemann theta function (see [9]). Then, as we mentioned in remark after Corollary 2, the algebro-geometric solution $\tau$ of the discrete KP equation becomes, with appropriate understanding of its argument via the divisor $D\left(n_{1}, n_{2}, n_{3}\right)$ and up to a not essential and non-vanishing multiplier, the Riemann theta function (see, e.g. [15]). In particular, in such an interpretation the zeros of $\tau$ are located in points of the translate $\mathbb{W}_{g-1}$ of the Theta divisor.

Let us discuss periodicity of solutions of the finite field version of the KP equation obtained using the above method. Denote by $\Pi_{i}, i=1,2,3$, the ranks of cyclic subgroups of $J(\mathcal{C} ; \mathbb{K})$ generated by divisors $A_{i}-A_{0}$, then for arbitrary $k_{i} \in \mathbb{Z}, i=$ $1,2,3$,

$$
D\left(n_{1}+k_{1} \Pi_{1}, n_{2}+k_{2} \Pi_{2}, n_{3}+k_{3} \Pi_{3}\right) \sim D\left(n_{1}, n_{2}, n_{3}\right) .
$$

In particular, $\tau\left(n_{1}, n_{2}, n_{3}\right)=0$ implies $\tau\left(n_{1}+k_{1} \Pi_{1}, n_{2}+k_{2} \Pi_{2}, n_{3}+k_{3} \Pi_{3}\right)=0$. 
There exist unique (normalized at $A_{0}$ ) functions $h_{i}, i=1,2,3$, with zeros of order $\Pi_{i}$ at $A_{i}$, poles of order $\Pi_{i}$ at $A_{0}$ and no other singularities and zeros such that

$$
\psi\left(n_{1}+k_{1} \Pi_{1}, n_{2}+k_{2} \Pi_{2}, n_{3}+k_{3} \Pi_{3}\right)=h_{1}^{k_{1}} h_{2}^{k_{2}} h_{3}^{k_{3}} \psi\left(n_{1}, n_{2}, n_{3}\right) .
$$

Remark. Generalizing above considerations, if for $\ell_{i} \in \mathbb{Z}, i=1,2,3$,

$$
\sum_{i=1}^{3} \ell_{i}\left(A_{i}-A_{0}\right) \sim 0,
$$

then $\left(\ell_{1}, \ell_{2}, \ell_{3}\right)$ is the period vector of zeros of the $\tau$-function and vector of quasiperiodicity (in the above sense) of the wave function.

Equation (8) implies quasi-periodicity of the functions $\zeta_{0}^{(i)}, i=1,2,3$,

$$
\zeta_{0}^{(i)}\left(n_{1}+k_{1} \Pi_{1}, n_{2}+k_{2} \Pi_{2}, n_{3}+k_{3} \Pi_{3}\right)=c_{(i) 1}^{k_{1}} c_{(i) 2}^{k_{2}} c_{(i) 3}^{k_{3}} \zeta_{0}^{(i)}\left(n_{1}, n_{2}, n_{3}\right),
$$

with the (non-zero) factors $c_{(i) j} \in \mathbb{K}_{*}$ equal to

$$
c_{(i) j}=\left.\left(\frac{h_{j}}{t_{j}^{\delta_{i j} \Pi_{j}}}\right)\right|_{P=A_{i}} .
$$

The multiplicative group $\mathbb{K}_{*}$ is a cyclic group of order $q-1$, therefore the functions $\zeta_{0}^{(i)}$ are periodic. Their periods in variable $n_{j}$ are equal to $\Pi_{j}$ times the order of the subgroup of $\mathbb{K}_{*}$ generated by $c_{(i) j}$ (a divisor of $q-1$ ). Due to possible change of sign (see equation (3) ) the periods of $\rho_{i}$ can be eventually doubled with respect to the corresponding periods of $\zeta_{0}^{(i)}$. Again, periodicity of $\rho_{i}$ implies quasi-periodicity of $\tau$ with a factor from $\mathbb{K}_{*}$, thus the period of $\tau$ in variable $n_{i}$ can be maximally $q-1$ times the period of $\rho_{i}$ in that variable.

\section{A "HYPERELliptid" SOLUtion OF The DisCRETE KP EQUATion}

Our goal here is to demonstrate how does the method described above work. We perform all steps of the construction (see also [2] for details) starting from a given algebraic curve, which we have chosen to be a hyperelliptic curve, due to relatively simple description of Jacobians of such curves [19]. We consider a hyperelliptic curve of genus $g=2$ but the technical tools used here can be applied directly to hyperelliptic curves of arbitrary genus.

3.1. A hyperelliptic curve and its Jacobian. Consider a hyperelliptic curve $\mathcal{C}$ of genus $g=2$ defined over the field $\mathbb{F}_{7}$ and given by the equation

$$
\mathcal{C}: v^{2}+u v=u^{5}+5 u^{4}+6 u^{2}+u+3 .
$$

The $(u, v)$ coordinates of its $\mathbb{F}_{7}$-rational points are presented in Table 1 The curve has one point at infinity, denoted by $\infty$, whose preimage on the nonsingular model of $\mathcal{C}$ consists of one point only [24], and where the local uniformizing parameter can be chosen as $u^{2} / v(u$ is a polynomial function of order 2 , and $v$ is a polynomial function of order 5). The point opposite (with respect to the hyperelliptic authomorphism) to $P$ is denoted by $\tilde{P}$. The only two special point of the curve are $(6,4)$ and the infinity point $\infty$. 


\begin{tabular}{|c||c|c|}
\hline \hline$i$ & $P_{i}$ & $\widetilde{P}_{i}$ \\
\hline \hline 0 & $\infty$ & $P_{0}$ \\
1 & $(1,1)$ & $(1,5)$ \\
2 & $(2,2)$ & $(2,3)$ \\
3 & $(5,3)$ & $(5,6)$ \\
4 & $(6,4)$ & $P_{4}$ \\
\hline \hline
\end{tabular}

TABLE $1 . \mathbb{F}_{7}$-rational points of the curve $\mathcal{C}$.

\begin{tabular}{|c||c|c|c|c|}
\hline \hline$i$ & $P_{i}$ & $\tilde{P}_{i}$ & $P_{i}^{\sigma}$ & $\tilde{P}_{i}^{\sigma}$ \\
\hline \hline 5 & $(0,21)$ & $(0,28)$ & $\tilde{P}_{5}$ & $P_{5}$ \\
6 & $(3,9)$ & $(3,44)$ & $\tilde{P}_{6}$ & $P_{6}$ \\
7 & $(4,26)$ & $(4,33)$ & $\tilde{P}_{7}$ & $P_{7}$ \\
\hline 8 & $(7,5)$ & $(7,44)$ & $(42,5)$ & $(42,9)$ \\
9 & $(8,22)$ & $(8,26)$ & $(43,29)$ & $(43,33)$ \\
10 & $(11,5)$ & $(11,47)$ & $(46,5)$ & $(46,12)$ \\
11 & $(12,6)$ & $(12,45)$ & $(47,6)$ & $(47,10)$ \\
12 & $(13,14)$ & $(13,29)$ & $(48,35)$ & $(48,22)$ \\
13 & $(14,8)$ & $(14,34)$ & $(35,43)$ & $(35,27)$ \\
14 & $(15,13)$ & $(15,28)$ & $(36,48)$ & $(36,21)$ \\
15 & $(16,17)$ & $(16,23)$ & $(37,38)$ & $(37,30)$ \\
16 & $(17,0)$ & $(17,39)$ & $(38,0)$ & $(38,18)$ \\
17 & $(18,4)$ & $(18,41)$ & $(39,4)$ & $(39,20)$ \\
18 & $(19,9)$ & $(19,28)$ & $(40,44)$ & $(40,21)$ \\
19 & $(20,12)$ & $(20,31)$ & $(41,47)$ & $(41,24)$ \\
20 & $(22,4)$ & $(22,30)$ & $(29,4)$ & $(29,23)$ \\
21 & $(25,6)$ & $(25,32)$ & $(32,6)$ & $(32,25)$ \\
22 & $(27,7)$ & $(27,22)$ & $(34,42)$ & $(34,29)$ \\
\hline \hline
\end{tabular}

TABLE $2 . \mathbb{F}_{49}$-rational points of the curve $\mathcal{C}$ (which are not $\mathbb{F}_{7}$-rational); here $\tilde{P}$ is the opposite of $P$, and $P^{\sigma}$ denotes its conjugate with respect to the lift of the Frobenius automorphism.

We identify the field $\mathbb{F}_{49}$ as the extension of $\mathbb{F}_{7}$ by the polynomial $x^{2}+2$, i.e., $\mathbb{F}_{49}=\mathbb{F}_{7}[x] /\left(x^{2}+2\right)$. Let us introduce the following notation: the element $k \in \mathbb{F}_{49}$ represented by the polynomial $\beta x+\alpha$ is denoted by the natural number $7 \beta+\alpha$. The Galois group $G\left(\mathbb{F}_{49} / \mathbb{F}_{7}\right)=\{\mathrm{id}, \sigma\}$, where $\sigma$ is the Frobenius automorphism, acts on elements of $\mathbb{F}_{49} \backslash \mathbb{F}_{7}$ in the following way:

$$
\mathbb{F}_{49} \backslash \mathbb{F}_{7} \ni k=7 \beta+\alpha \mapsto \sigma(k)=7(7-\beta)+\alpha .
$$

The coordinates of $\mathbb{F}_{49}$-rational points of the curve (which are not $\mathbb{F}_{7}$-rational) are presented in Table 2. 
In the next step we find the group of the $\mathbb{F}_{7}$-rational points $J\left(\mathcal{C} ; \mathbb{F}_{7}\right)$ of the Jacobian of the curve. The number of its points can be found from the number of $\mathbb{F}_{7}$-rational and $\mathbb{F}_{49}$-rational points of the curve by application of properties of the zeta function of the curve $\mathcal{C}$ (see for instance [25, 13]). In our case the curve has $8 \mathbb{F}_{7}$-rational points and $74 \mathbb{F}_{49}$-rational points which implies the following form of the zeta function $\zeta(\mathcal{C} ; T)$

$$
\zeta(\mathcal{C} ; T)=\frac{P(T)}{(1-T)(1-7 T)}, \quad P(T)=1+12 T^{2}+49 T^{4} .
$$

The number $\# J\left(\mathcal{C} ; \mathbb{F}_{7}\right)$ of the $\mathbb{F}_{7}$-rational points points of the Jacobian is equal to $P(1)=62$, and therefore $J\left(\mathcal{C} ; \mathbb{F}_{7}\right)$ is the direct sum of cyclic groups of orders 31 and 2.

Let us choose the infinity point $\infty$ as the basepoint. The group law in the Jacobian of a hyperelliptic curve can be intuitively described in a way which is a higher-genus analog of the well known addition operation for points of elliptic curves. We present here only its sketch for genus $g=2$ and in the generic case of addition of two points of $J(\mathcal{C})$ with representants of the form

$$
E_{i}=Q_{i}+R_{i}-2 \infty, \quad i=1,2,
$$

with all points distinct. If $E_{3}=Q_{3}+R_{3}-2 \infty$ is the representant of $\left[E_{1}+E_{2}\right]$, i.e.,

$$
E_{1}+E_{2}=(g)+E_{3},
$$

then

$$
E_{1}+E_{2}+\tilde{E}_{3} \sim 0
$$

where we have used the fact that for any point $P \in \mathcal{C}(\overline{\mathbb{K}})$ of a hyperelliptic curve the divisor $P+\tilde{P}-2 \infty$ is principal. Therefore, there exists a normalized polynomial function $f$ of the order six, thus necessarily of the form

$$
f=a+b u+c u^{2}+d u^{3}+e v,
$$

with divisor given by the left hand side of equation (10). Its zeros at $Q_{i}$ and $R_{i}$, $i=1,2$, and the normalization condition allow to fix the coefficients and then to find two other zeros $\tilde{Q}_{3}$ and $\tilde{R}_{3}$.

Geometrically, we are looking for two other intersection points of the cubic interpolating known four points with the hyperelliptic curve. In cases when some points of $E_{1}+E_{2}$ are repeated, the interpolation step must be adjusted to ensure tangency to the curve with sufficient multiplicity. When divisors have less points then we consider the interpolating curve of lower degree (some intersection points are at infinity). Finally, the transition function $g$ is the unique normalized function with the nominator equal to $f$ and the denominator being the normalized polynomial function with the divisor $E_{3}+\tilde{E}_{3}$.

The full description of the group $J\left(\mathcal{C} ; \mathbb{F}_{7}\right)$ is given in Table 3 . The divisor $D_{1}=$ $P_{1}-\infty$ generates the subgroup of order 31 and the divisor $D_{4}=P_{4}-\infty$ generates the subgroup of order 2 . We present the reduced representants $\left[n D_{1}+m D_{4}\right]_{r}$ of elements $\left[n D_{1}+m D_{4}\right]$ of $J\left(\mathcal{C} ; \mathbb{F}_{7}\right)$, where $n \in\{0,1, \ldots, 30\}$ and $m \in\{0,1\}$. Moreover we write down the transition functions $g_{m}(n)$ defined by the following divisor equation

$$
\left[n D_{1}+m D_{4}\right]_{r}+D_{1}=\left(g_{m}(n)\right)+\left[(n+1) D_{1}+m D_{4}\right]_{r} .
$$


Also the transition functions for the sums $\left[n D_{1}+m D_{4}\right]_{r}+D_{4}$ can be read off from Table 3. In particular, to find such a transition function (we call it $W(0,1)$ ) with $n=29$ and $m=0$, i.e.,

$$
(1,5)+(1,5)+D_{4}-2 \infty=(W(0,1))+(12,6)+(47,6)-2 \infty
$$

we make use of the fact that the analogous transition function for $n=30$ and $m=0$ is 1 . Then

$$
W(0,1)=g_{0}(29) \cdot 1 \cdot\left[g_{1}(29)\right]^{-1}=\frac{2+3 u+4 u^{2}+v}{6+4 u+u^{2}},
$$

where in the last equality we have used equation (9) of the curve to get rid of $v$ from the denominator.

3.2. Construction of the wave and $\tau$ functions. In order to find a solution of the discrete KP equation let us fix the following points of the curve $\mathcal{C}$,

$$
A_{0}=\infty, \quad A_{1}=(1,1), \quad A_{2}=(2,2), \quad A_{3}=(5,3),
$$

with the uniformizing parameters

$$
t_{0}=u^{2} / v, \quad t_{1}=u-1, \quad t_{2}=u-2, \quad t_{3}=u-5
$$

and

$$
B_{1}=(12,6), \quad B_{2}=(47,6)
$$

Because

$$
\begin{array}{cl}
A_{0}-A_{1} \sim 30 D_{1}, & A_{0}-A_{2} \sim 21 D_{1}+D_{4}, \quad A_{0}-A_{3} \sim 17 D_{1}+D_{4}, \\
& B_{1}+B_{2}-2 A_{0} \sim 29 D_{1}+D_{4},
\end{array}
$$

then the points $X_{1}\left(n_{1}, n_{2}, n_{3}\right)$ and $X_{2}\left(n_{1}, n_{2}, n_{3}\right)$ where the wave function $\psi\left(n_{1}, n_{2}, n_{3}\right)$ has additional zeros can be found from Table 3 and from equation

$$
X_{1}\left(n_{1}, n_{2}, n_{3}\right)+X_{2}\left(n_{1}, n_{2}, n_{3}\right)-2 \infty=\left[n D_{1}+m D_{4}\right]_{r},
$$

where $n \in\{0,1, \ldots, 30\}$ and $m \in\{0,1\}$ are given by

$$
\begin{aligned}
& \left.n=29+30 n_{1}+21 n_{2}+17 n_{3}\right) \quad \bmod 31, \\
& m=1+n_{2}+n_{3} \bmod 2 .
\end{aligned}
$$

Remark. Notice that the infinity point $\infty$ is the Weierstrass point of the curve $\mathcal{C}$, which violates the assumption of general position of points used in the construction of solutions of the discrete KP equation. This will not change the Jacobian picture of the construction but in some situations, which we will point out, will affect uniqueness of the wave function. We remark that such a choice is indispensable in reduction of the method from the discrete KP equation to the discrete KdV equation (see, for example [15, 3]).

To find effectively the wave function we will constraint the range of parameters from $\mathbb{Z}^{3}$ to the parameters of the group of $\mathbb{F}_{7}$-rational points of the Jacobian. Let us introduce functions $h_{1}$ and $h_{4}$ corresponding to generators of the two cyclic subgroups 


\begin{tabular}{|c|c|c|c|c|}
\hline$n$ & {$\left[n D_{1}\right]_{r}$} & $g_{0}(n)$ & {$\left[n D_{1}+D_{4}\right]_{r}$} & $g_{1}(n)$ \\
\hline 0 & 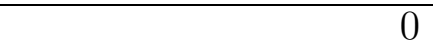 & 1 & $(6,4)-\infty$ & 1 \\
\hline 1 & $(1,1)-\infty$ & 1 & $(1,1)+(6,4)-2 \infty$ & $\frac{5+5 u+3 u^{2}+v}{6+4 u+u^{2}}$ \\
\hline 2 & $(1,1)+(1,1)-2 \infty$ & $\frac{u+5 u^{2}+v}{(2+u)^{2}}$ & $(12,45)+(47,10)-2 \infty$ & $\frac{1+5 u^{2}+v}{2+5 u+u^{2}}$ \\
\hline 3 & $(5,6)+(5,6)-2 \infty$ & $\frac{1+u+4 u^{2}+v}{(2+u)(5+u)}$ & $(15,28)+(36,21)-2 \infty$ & $\frac{6 u^{2}+v}{2+u^{2}}$ \\
\hline 4 & $(2,3)+(5,3)-2 \infty$ & $\frac{2+4 u^{2}+v}{5+4 u+u^{2}}$ & $(7,44)+(42,9)-2 \infty$ & $\frac{5+u+v}{4+6 u+u^{2}}$ \\
\hline 5 & $(19,9)+(40,44)-2 \infty$ & $\frac{4 u+2 u^{2}+v}{5+5 u+u^{2}}$ & $(11,5)+(46,5)-2 \infty$ & $\frac{6+6 u+u^{2}+v}{3+6 u+u^{2}}$ \\
\hline 6 & $(22,4)+(29,4)-2 \infty$ & $\frac{5+2 u+6 u^{2}+v}{(2+u)(5+u)}$ & $(18,41)+(39,20)-2 \infty$ & $\frac{5+3 u+5 u^{2}+v}{5+3 u+u^{2}}$ \\
\hline 7 & $(2,3)+(5,6)-2 \infty$ & $\frac{5+6 u+2 u^{2}+v}{5+2 u+u^{2}}$ & $(16,17)+(37,38)-2 \infty$ & $\frac{5+4 u+4 u^{2}+v}{3+u+u^{2}}$ \\
\hline 8 & $(27,22)+(34,29)-2 \infty$ & $\frac{1+3 u+2 u^{2}+v}{1+u^{2}}$ & $(17,39)+(38,18)-2 \infty$ & $\frac{3+2 u+u^{2}+v}{(5+u)(6+u)}$ \\
\hline 9 & $(14,34)+(35,27)-2 \infty$ & $\frac{1+5 u+v}{(1+u)(5+u)}$ & $(1,5)+(2,2)-2 \infty$ & $6+u$ \\
\hline 10 & $(2,2)+(6,4)-2 \infty$ & $\frac{3+5 u+5 u^{2}+v}{(5+u)^{2}}$ & $(2,2)-\infty$ & 1 \\
\hline 11 & $(2,3)+(2,3)-2 \infty$ & $\frac{6+u+6 u^{2}+v}{3+2 u+u^{2}}$ & $(1,1)+(2,2)-2 \infty$ & $\frac{4+2 u^{2}+v}{3+5 u+u^{2}}$ \\
\hline 12 & $(13,14)+(48,35)-2 \infty$ & $\frac{3+6 u+4 u^{2}+v}{2+2 u+u^{2}}$ & $(8,22)+(43,29)-2 \infty$ & $\frac{2+4 u+v}{(2+u)(6+u)}$ \\
\hline 13 & $(20,12)+(41,47)-2 \infty$ & $\frac{5 u+u^{2}+v}{(1+u)(2+u)}$ & $(1,5)+(5,3)-2 \infty$ & $6+u$ \\
\hline 14 & $(5,3)+(6,4)-2 \infty$ & $\frac{6+5 u+2 u^{2}+v}{6+6 u+u^{2}}$ & $(5,3)-\infty$ & 1 \\
\hline 15 & $(25,32)+(32,25)-2 \infty$ & $\frac{5+u^{2}+v}{6+6 u+u^{2}}$ & $(1,1)+(5,3)-2 \infty$ & $\frac{u+5 u^{2}+v}{(2+u)(6+u)}$ \\
\hline 16 & $(25,6)+(32,6)-2 \infty$ & $\frac{6+5 u+2 u^{2}+v}{(1+u)(2+u)}$ & $(1,5)+(5,6)-2 \infty$ & $6+u$ \\
\hline 17 & $(5,6)+(6,4)-2 \infty$ & $\frac{5 u+u^{2}+v}{2+2 u+u^{2}}$ & $(5,6)-\infty$ & 1 \\
\hline 18 & $(20,31)+(41,24)-2 \infty$ & $\frac{3+6 u+4 u^{2}+v}{3+2 u+u^{2}}$ & $(1,1)+(5,6)-2 \infty$ & $\frac{2+4 u+v}{3+5 u+u^{2}}$ \\
\hline 19 & $(13,29)+(48,22)-2 \infty$ & $\frac{u^{2}+v}{e^{2}}$ & $(8,26)+(43,33)-2 \infty$ & $\frac{4+2 u^{2}+v}{(5+u)(6+u)}$ \\
\hline 20 & $(2,2)+(2,2)-2 \infty$ & $\frac{3+5 u+5 u^{2}+v}{(1+u)(5+u)}$ & $(1,5)+(2,3)-2 \infty$ & $6+u$ \\
\hline 21 & $(2,3)+(6,4)-2 \infty$ & $\frac{1++5 u+v}{1+u^{2}}$ & $(2,3)-\infty$ & 1 \\
\hline 22 & $(14,8)+(35,43)-2 \infty$ & $\frac{1+3 u+2 u^{2}+v}{5+2 u+u^{2}}$ & $(1,1)+(2,3)-2 \infty$ & $\frac{3+2 u+u^{2}+v}{3+u+u^{2}}$ \\
\hline 23 & $(27,7)+(34,42)-2 \infty$ & $\frac{5+6 u+2 u^{2}+v}{(2+u)(5+u)}$ & $(17,0)+(38,0)-2 \infty$ & $\frac{5+4 u+4 u^{2}+v}{5+3 u+u^{2}}$ \\
\hline 24 & $(2,2)+(5,3)-2 \infty$ & $\frac{6 u^{2}+v}{+u^{2}}$ & $(16,23)+(37,30)-2 \infty$ & $\frac{5+3 u+5 u^{2}+v}{3+6 u+u^{2}}$ \\
\hline 25 & $(22,30)+(29,23)-2 \infty$ & $\frac{t^{2}+v}{+u^{2}}$ & $(18,4)+(39,4)-2 \infty$ & $\frac{6+6 u+u^{2}+v}{4+6 u+u^{2}}$ \\
\hline 26 & $(19,28)+(40,21)-2 \infty$ & $\frac{2+4 u^{2}+v}{(2+u)(5+u)}$ & $(11,47)+(46,12)-2 \infty$ & $\frac{5+u+v}{2+u^{2}}$ \\
\hline 27 & $(2,2)+(5,6)$ & $\frac{1+u+4 u^{2}+v}{(2+u)^{2}}$ & $(7,5)+(42,5)-2 \infty$ & $\frac{6 u^{2}+v}{2+5 u+u^{2}}$ \\
\hline 28 & $(5,3)+(5,3)-2 \infty$ & $\frac{u+5 u^{2}+v}{(6+u)^{2}}$ & $(15,13)+(36,48)-2 \infty$ & $\frac{1+5 u^{2}+v}{6+4 u+u^{2}}$ \\
\hline 29 & $(1,5)+(1,5)-2 \infty$ & $(6+u)$ & $(12,6)+(47,6)-2 \infty$ & $\frac{5+5 u+3 u^{2}+v}{(1+u)(6+u)}$ \\
\hline 30 & $(1,5)-\infty$ & $(6+u)$ & $(1,5)+(6,4)-2 \infty$ & $6+u$ \\
\hline
\end{tabular}

TABLE 3. The group $J\left(\mathcal{C} ; \mathbb{F}_{7}\right)$ of $\mathbb{F}_{7}$-rational points of the Jacobian as the simple sum of its cyclic subgroups. 
of $J\left(\mathcal{C} ; \mathbb{F}_{7}\right)$. The function $h_{1}$ with the divisor $31 D_{1}$ and normalized at the infinity point is equal to the product $\prod_{i=0}^{30} g_{0}(i)$ and reads

$$
\begin{gathered}
h_{1}=1+2 u+u^{2}+4 u^{3}+3 u^{5}+u^{6}+3 u^{7}+u^{8}+4 u^{9}+ \\
4 u^{10}+2 u^{11}+5 u^{12}+2 u^{13}+4 u^{14}+3 u^{15}+\left(5 u+2 u^{2}+\right. \\
\left.5 u^{3}+4 u^{5}+6 u^{6}+4 u^{7}+3 u^{9}+5 u^{10}+5 u^{11}+4 u^{12}+u^{13}\right) v
\end{gathered}
$$

where we also used equation of the curve (9) to reduce higher order terms in $v$. The normalized function $h_{4}$ with the divisor $2 D_{4}$ is

$$
h_{4}=u-6 .
$$

Let us introduce other auxiliary functions $f_{2}$ and $f_{3}$ to factorize the zeros of the wave function at $A_{2}$ and $A_{3}$. Notice that

$$
A_{2}+21 D_{1}+D_{4}-\infty \sim 0
$$

which implies that there exists a polynomial function on $\mathcal{C}$ with simple zero at $A_{2}$ and other zeros in the distinguished (by our choice of description of $J\left(\mathcal{C} ; \mathbb{F}_{7}\right)$ ) points $(1,1)$ and $(6,4)$. Define $f_{2}$ as the unique such function normalized at the infinity point $\infty$, then

$$
\begin{aligned}
f_{2}=1+5 u+u^{2}+4 u^{4}+6 u^{5}+4 u^{6}+4 u^{7}+3 u^{8}+4 u^{9}+ \\
6 u^{11}+\left(6+4 u+2 u^{2}+5 u^{3}+6 u^{4}+6 u^{6}+u^{7}+u^{8}+u^{9}\right) v
\end{aligned}
$$

Similarly we define the normalized function

$f_{3}=1+6 u+2 u^{2}+6 u^{5}+u^{6}+5 u^{7}+5 u^{8}+4 u^{9}+\left(4+3 u+5 u^{2}+4 u^{5}+2 u^{6}+u^{7}\right) v$ with the divisor $A_{3}+17 D_{1}+D_{4}-\infty$.

Uniqueness of the wave function $\psi$ implies that it can be decomposed as follows

$$
\psi\left(n_{1}, n_{2}, n_{3}\right)=\frac{f_{2}^{n_{2}} f_{3}^{n_{3}}}{h_{1}^{p} h_{4}^{q}} W\left(m_{1}, m_{2}\right),
$$

where $W\left(m_{1}, m_{2}\right)$ is the unique normalized function with the divisor

$$
m_{1} D_{1}+m_{2} D_{4}+Y_{1}\left(m_{1}, m_{2}\right)+Y_{2}\left(m_{1}, m_{2}\right)-(12,6)-(47,6)
$$

where

$$
Y_{1}\left(m_{1}, m_{2}\right)+Y_{2}\left(m_{1}, m_{2}\right)=X_{1}\left(n_{1}, n_{2}, n_{3}\right)+X_{2}\left(n_{1}, n_{2}, n_{3}\right),
$$

and the new variables $m_{1}$ i $m_{2}$ are given by

$$
\begin{aligned}
21 n_{2}+17 n_{3}-n_{1} & =31 p-m_{1}, \quad m_{1} \in\{0,1, \ldots, 30\}, \\
n_{2}+n_{3} & =2 q-m_{2}, \quad m_{2} \in\{0,1\} .
\end{aligned}
$$

To find the functions $W\left(m_{1}, m_{2}\right)$ for all $m_{1} \in\{0,1, \ldots, 30\}$ and $m_{2} \in\{0,1\}$ let us first notice that $W(0,0)=1$ and $W(0,1)$ is indeed the function found in equation (13). For $m_{1} \in\{1, \ldots, 30\}$ and $m_{2} \in\{0,1\}$ define the functions $w_{m_{2}}\left(m_{1}\right)$ as follows

$$
W\left(m_{1}, m_{2}\right)=w_{m_{2}}\left(m_{1}\right) W\left(m_{1}-1, m_{2}\right) .
$$


Equations (11), (14)-(16) and (18)-(21) imply that for such range of $m_{1}$ and $m_{2}$ we have

$$
g_{m}(n)=w_{m_{2}}\left(m_{1}\right)
$$

where

$$
m_{2}=1-m \quad \bmod 2, \quad m_{1}=29-n \bmod 31 .
$$

Finally, under identification $w_{m_{2}}(0)=W\left(0, m_{2}\right)$ we obtain

$$
W\left(m_{1}, m_{2}\right)=\prod_{i=0}^{m_{1}} w_{m_{2}}(i),
$$

which, together with factorization (17), gives the wave function $\psi$ for all values of $\left(n_{1}, n_{2}, n_{3}\right) \in \mathbb{Z}^{3}$.

Remark. For $\left(m_{1}, m_{2}\right)=(29,1)$ we have $X_{1}=X_{2}=\infty$. Because the infinity point $\infty$ is the Weierstrass point of order two, there exist functions with divisor of poles equal to $2 \infty$. This means that $\psi$ is not uniqely determined in this case. However it is natural to keep the divisor of $\psi$, and therefore $\psi$ itself, exactly like it is given from the flow on Jacobian. Notice that because for $X_{1}=X_{2}=\infty$ we stay in the divisor $\mathbb{W}_{g-1}$ then this ambiguity does not affect construction of the $\tau$-function.

The coefficients $\zeta_{0}{ }^{(k)}\left(n_{1}, n_{2}, n_{3}\right), k=1,2,3$, of expansion of the wave function can be obtained from factorization (17) and are given by

$$
\begin{aligned}
& \zeta_{0}^{(1)}\left(n_{1}, n_{2}, n_{3}\right)=\left.6^{n_{3}+p} 4^{q}\left(\frac{W\left(m_{1}, m_{2}\right)}{t_{1}^{m_{1}}}\right)\right|_{t_{1}=0}, \\
& \zeta_{0}^{(2)}\left(n_{1}, n_{2}, n_{3}\right)=\left.6^{n_{2}} 5^{n_{3}+q} 4^{p} W\left(m_{1}, m_{2}\right)\right|_{t_{2}=0}, \\
& \zeta_{0}^{(3)}\left(n_{1}, n_{2}, n_{3}\right)=\left.5^{n_{2}+n_{3}} 3^{p} 6^{q} W\left(m_{1}, m_{2}\right)\right|_{t_{3}=0} .
\end{aligned}
$$

Using definition of the $\tau$ function for nonzero $\rho_{i}$, i.e. equation (4), and putting $\tau=0$ for points of the divisor $\mathbb{W}_{g-1}$ we obtain the corresponding $\mathbb{F}_{7}$-valued solution of the discrete KP equation (6). This $\tau$-function is presented in Figure 1. Notice that due to quasi-periodicity of the $\tau$-function, we have to calculate the solution in this way only for a finite range of values of the variables.

The periods $\Pi_{i}, i=1,2,3$, of zeros of the $\tau$-function are, respectively, 31, 62 and 62. Equivalently, the "period vectors" of zeros can be choosen as

$$
v_{1}=\left(\begin{array}{c}
-4 \\
-1 \\
1
\end{array}\right) \quad v_{2}=\left(\begin{array}{c}
11 \\
2 \\
0
\end{array}\right) \quad v_{3}=\left(\begin{array}{l}
2 \\
6 \\
0
\end{array}\right)
$$

Because $c_{(1) 1}=6, c_{(2) 1}=3$ and $c_{(3) 1}=5$ then periods of the functions $\rho_{i}, i=1,2,3$, in that variable are, respectively, $2 \cdot 31,3 \cdot 31 \cdot 2$ and $6 \cdot 31$. Moreover, we have $\tau(31,0,0)=3$ which gives $\tau(62,0,0)=5=3^{2} \cdot 6^{31} \bmod 7$, and therefore the period of $\tau$ in $n_{1}$ is $6 \cdot 2 \cdot 31$.

\section{ACKNOWLEDGMENTS}

The paper was partially supported by the University of Warmia and Mazury in Olsztyn under the grant 522-1307-0201 and by KBN grant 2 P03B 12622. 

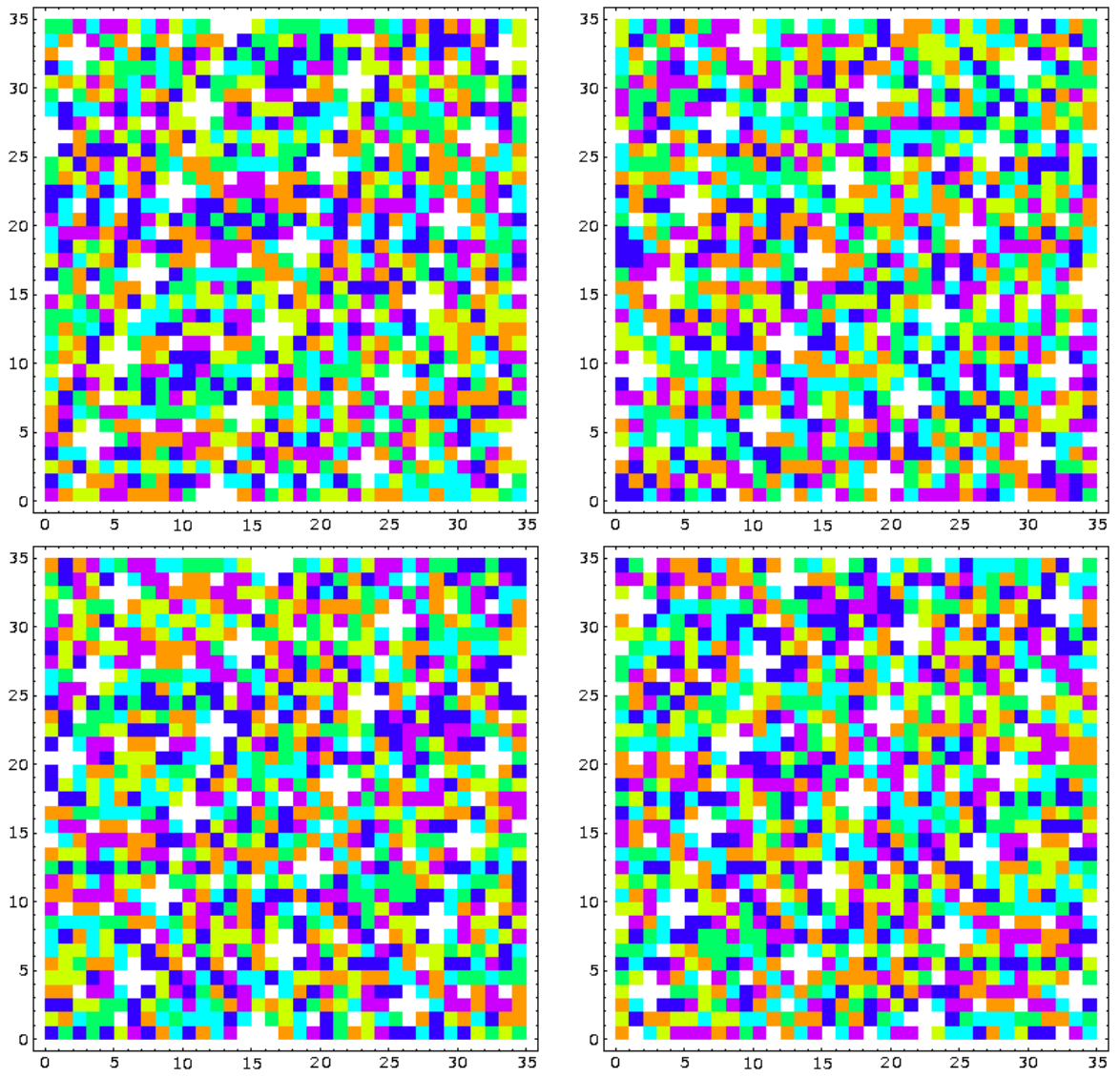

$\square-0, \square-1, \square-2, \square-3, \square-4, \square-5, \square-6$.

FiguRE $1 . \mathbb{F}_{7}$-valued solutions of discrete KP equation out of genus two hiperelliptic curve $\mathcal{C}$. Variables $n_{1}$ (directed to the right) and $n_{2}$ (directed up) take values from 0 to 34 . Subsequent figures are for values of $n_{3}=-1,0,1,2$.

\section{REFERENCES}

1. E. D. Belokolos, A. I. Bobenko, V. Z. Enol'skii, A. R. Its, and V. B. Matveev, Algebro-geometric approach to nonlinear integrable equations, Springer-Verlag, Berlin, 1994.

2. M. Białecki, Methods of algebraic geometry over finite fields in construction of integrable cellular automata, $\mathrm{PhD}$ dissertation, Warsaw University, Institute of Theoretical Physics, 2003.

3. M. Białecki and A. Doliwa, The discrete KP and KdV equations over finite fields, Theor. Math. Phys. 137 (2003), 1410-1416.

4. A. Bobenko, M. Bordemann, Ch. Gunn, and U. Pinkall, On two integrable cellular automata, Comm. Math. Phys. 158 (1993), 127-134.

5. M. Bruschi and P. M. Santini, Cellular automata in $1+1,2+1$ and $3+1$ dimensions, constants of motion and coherent structures, Physica D 70 (1994), 185-209.

6. G. Cornell and J. H. Silverman (eds.), Arithmetic geometry, Springer-Verlag, New York, 1986.

7. A. Doliwa, M. Białecki, and P. Klimczewski, The Hirota equation over finite fields: algebrogeometric approach and multisoliton solutions, J. Phys. A 36 (2003), 4827-4839. 
8. A.S. Fokas, E.P. Papadopoulou, and Y.G. Saridakis, Soliton cellular automata, Physica D 41 (1990), 297-321.

9. P. Griffiths and J. Harris, Principles of algebraic geometry, John Wiley and Sons, New York, 1978.

10. R. Hartshorne, Algebraic geometry, Springer-Verlag, New York, 1977.

11. R. Hirota, Nonlinear partial difference equations. III. Discrete sine-Gordon equation, J. Phys. Soc. Jpn. 43 (1977), 2079-2086.

12. _ Discrete analogue of a generalized Toda equation, J. Phys. Soc. Jpn. 50 (1981), 37853791.

13. N. Koblitz, Algebraic aspects of cryptography, Springer-Verlag, Berlin, 1998.

14. I. M. Krichever, Algebraic curves and non-linear difference equations, Uspiekhi Mat. Nauk 33 (1978), 215-216.

15. I. M. Krichever, P. Wiegmann, and A. Zabrodin, Elliptic solutions to difference non-linear equations and related many body problems, Commun. Math. Phys. 193 (1998), 373-396.

16. S. Lang, Abelian varieties, Interscience Publishers, Inc., New York, 1958.

17. Algebra, Addison-Wesley, Reading, Mass., 1970.

18. J. Matsukidaira, J. Satsuma, D. Takahashi, T. Tokihiro, and M. Torii, Toda-type cellular automaton and its $N$-soliton solution, Phys. Lett. A 225 (1997), 287-295.

19. A. J. Menezes, Y. H. Wu, and R. J. Zuccherato, An elementary introduction to hyperelliptic curves, appendix in [13, pp. 151-178.

20. J. S. Milne, Jacobian varieties, Chapter VII in [6, pp. 167-212.

21. C. Moreno, Algebraic curves over finite fields, University Press, Cambridge, 1991.

22. D. Mumford, An algebro-geometric construction of commuting operators and of solutions to the Toda lattice equation, Korteweg-de Vries equation, and related nonlinear equations, Proceedings of the International Symposium on Algebraic Geometry (M. Nagata, ed.), Kinokuniya, Tokyo, 1978, pp. 115-153.

23. J. von Neumann, The general and logical theory of automata, The collected works of John von Neumann (A. W. Taub, ed.), vol. 5, Pergamon Press, 1963.

24. I. Shafarevich, Basic algebraic geometry, Springer-Verlag, Heidelberg, 1974.

25. H. Stichtenoth, Algebraic function fields and codes, Springer-Verlag, Berlin, 1993.

26. D. Takahashi and J. Satsuma, A soliton cellular automaton, J. Phys. Soc. Jpn. 59 (1990), 35143519.

27. T. Tokihiro, D. Takahashi, J. Matsukidaira, and J. Satsuma, From soliton equations to integrable cellular automata through a limiting procedure, Phys. Rev. Lett. 76 (1996), 3247-3250.

28. S. Ulam, Random processes and transformations, Proceedings of the International Congress of Mathematicians, Cambridge, MA, 30 August-6 September 1950 (P. A. Smith and O. Zariski, eds.), AMS, Providence, 1952, pp. 264-275.

29. S. Wolfram, Theory and application of cellular automata, World Scientific, Singapore, 1986.

M. Biąecki, Instytut Geofizyki PAN, ul. Ksiȩcia Janusza 64, 01-452 WarszaWA, Poland

E-mail address: bialecki@igf .edu.pl

A. Doliwa, Wydzią Matematyki i Informatyki, Uniwersytet Warmińsko-Mazurski, Ul. Żolnierska 14A, 10-561 OlszTyn, Poland

E-mail address: doliwa@matman.uwm.edu.pl 